\title{
:
}

\section{LA INTERNACIONALIZACIÓN DE LA PUBLICACIÓN COLOMBIANA EN EL CAMPO DE LA ADMINISTRACIÓN: AVANCES Y DESAFÍOS*}

\author{
Florentino Malaver Rodríguez**
}

* doi: 10.11144/Javeriana.cao29-52.ipcc. El autor agradece los comentarios y aportes realizados por los dos evaluadores anónimos. El artículo se recibió el 19/02/2016 y se aprobó el 15/06/2016. Sugerencia de citación: Malaver, F. (2016). La internacionalización de la publicación colombiana en el campo de la administración: avances y desafíos. Cuadernos de Administración, 29 (52), 53-81. http://dx.doi.org/10.11144/Javeriana. cao29-52.ipcc.

** $\mathrm{PhD}(\mathrm{c})$ en Competitividad Empresarial y Desarrollo Económico, Universidad de Deusto, España. Magister en Economía de la Universidad Nacional de Colombia. Profesor Asociado de la Facultad de Ciencias Económicas y Administrativas, Pontificia Universidad Javeriana, Bogotá, Colombia.

Correo electrónico: fmalaver@javeriana.edu.co 


\section{La internacionalización de la publicación colombiana en el campo de la administración: avances y desafios}

\section{Resumen}

Las facultades de administración colombianas (FCA) enfrentan una "presión institucional" creciente para incrementar la cantidad y calidad de su publicación internacional. El propósito del texto es establecer cuál ha sido la respuesta de las FCA a esa presión y analizar alternativas para enfrentar los desafíos que plantea dicha internacionalización. La indagación sistemática en la WoS y Scopus entre 2001 y 2015 revela un proceso de internacionalización en ciernes, que es simultáneo al registrado en otros países latinoamericanos. La discusión internacional sobre la publicación en management abre posibilidades que implican asumir la realidad propia como reservorio de oportunidades para generar conocimiento original y desarrollar indicadores que suplan las limitaciones de la citación para captar la utilidad-social-de los artículos.

Palabras clave: investigación, management, relevancia, citación, administración, Colombia.

Clasificación JEL: M19

\section{The internationalization of the Colombian publication in the field of management: Advances and challenges}

\section{ABSTRACT}

The Colombian management faculties (FCA) face a growing "institutional pressure" to increment the quantity and quality of their international publication. The purpose of this text is to establish how the FCA have answered to such pressure and analyze alternatives to face the challenges that this internationalization has posed. The systematic investigation in the Wos and Scopus between 2001 and 2015 reveals an internationalization process that is in its infancy, which is simultaneous to the process registered in other Latin American countries. The international discussion on the management publication opens possibilities that require assuming reality itself as a reservoir of opportunities to generate original knowledge and develop indicators that can supply the quotation limitations to understand the -social- utility of the articles.

Keywords: research, management, relevance, quotation, administration, Colombia.

JEL Classification: M19

\section{A internacionalização da publicação colombiana no campo da administração: avanços e desafios}

\section{RESUMO}

As faculdades de administração colombianas (FCA) enfrentam uma "pressão institucional" crescente para aumentar a quantidade e qualidade de sua publicação internacional. O propósito do texto é estabelecer qual foi a resposta das FCA a essa pressão e analisar alternativas para enfrentar os desafios que propõe essa internacionalização. A indagação sistemática na WoS e Scopus entre 2001 e 2015 revela um processo de internacionalização no início, que é simultâneo ao registrado em outros países latino-americanos. A discussão internacional sobre a publicação em management abre possibilidades que implicam assumir a realidade própria como reservatório de oportunidades para gerar conhecimento original e desenvolver indicadores que atendam as limitações da citação para captar a utilidade-social-dos artigos.

Palavras-chave: pesquisa, management, relevância, citação, administração, Colômbia.

Classificação JEL: M19 


\section{Introducción}

Luego de tres décadas en que sucesivos estudios diagnosticaron un estado incipiente de la investigación sobre la administración en Colombia, en 2006 se identificó un incremento de las publicaciones, inducido por una política pública que propició la institucionalización de la actividad investigativa en las facultades de administración colombianas (FCA) y el "despegue de la investigación" en administración en el país (Malaver, 2006). Ese proceso luego dio paso a una oleada de creación de programas de doctorado (Calderón et al., 2010) y a una fuerte "presión institucional" para aumentar la publicación internacional de calidad en las FCA (Orozco, 2015). En esa presión confluyeron la presión de la política pública en cabeza de Colciencias, los rankings -que clasifican internacional y nacionalmente a universidades, facultades e, incluso, MBAs- y las acreditadoras internacionales a las que acuden cada vez más FCA en busca de acreditaciones que mejoren su posicionamiento académico. Para todos ellos la publicación internacional de su cuerpo profesoral constituye un indicador fundamental para evaluar las facultades. A esto se sumó el acelerado aumento de las exigencias para la evaluación de las publicaciones que, de referentes nacionales consignados en el Publindex, transitó a criterios y estándares internacionales cada vez más exigentes, como el cuartil de las revistas y el impacto -por las citaciones recibidas-. Esto plantea desafíos descomunales para las FCA, de cuya respuesta dependerá el futuro de la investigación en el ámbito de la administración en Colombia.

Por ello este trabajo se propone identificar y evaluar: $i$ ) la respuesta de las FCA a la presión institucional para la publicación internacional, mediante una revisión sistemática de la cantidad y calidad de las publicaciones efectuadas en los journals internacionales, incorporados en las bases de datos bibliográficas más importantes del mundo; ii) las principales tensiones y desafíos que conlleva aumentar la publicación con la calidad exigida, y explorar en busca de alternativas -enmarcadas en la discusión internacional en el campo del management- para enfrentarlos de modo que aumente la publicabilidad y la relevancia del conocimiento generado en las FCA.

\section{Un marco contextual para evaluar los avances, las tensiones y los desafíos de la publicación internacional de las FCA}

Este trabajo intenta dar continuidad a la indagación por la actividad investigativa en el campo de la administración en Colombia, realizada por algo más de tres décadas. En esos trabajos pueden identificarse un primer momento, iniciado en la década de 1980 con las indagaciones de Dávila $(1980,1986)$ y Ogliastri (1982), que es continuado con los 
estudios de la década de 1990 por Rodríguez et al. (1992) y Malaver (2000), y en el que coincidían en que la investigación estaba en estado incipiente. Éste se había convertido en un estado endémico. Un segundo momento y punto de inflexión es inducido por la política pública -agenciada por Colciencias-, que impulsa la construcción de capacidades para producir y divulgar conocimiento -mediante el impulso a los grupos de investigación y a las revistas académicas- también por exigencias e incentivos normativos, como ligar por parte del Ministerio de educación la investigación a la evaluación y acreditación de programas de pregrado y posgrado, o establecer puntajes para las publicaciones de investigadores de las universidades públicas, con efectos salariales o de escalafón, de acuerdo con la categoría de indexación de las revistas donde publican. Estas exigencias normativas junto con los incentivos incidieron en un aumento acelerado en la producción académica publicada en el país en sus distintas disciplinas (Malaver y Vargas, 2005), que se extiende al área de la administración (Malaver, 2006).

Tales políticas provocan la generación de condiciones para la institucionalización de la investigación al nivel de cada FCA, para convertirse en una práctica individual y colectiva recurrente que genera aprendizajes que se acumulan, enriquecen y propician el aumento de la producción académica y de una cultura académica. Esto, más el notable incremento de las publicaciones, permiten concluir a Malaver (2006) que en la primera década del nuevo siglo se produjo el "despegue" de la investigación en administración. Ese diagnóstico es reforzado por trabajos posteriores que consideran que en las FCA se cuenta con grupos de investigación de élite (Calderón et al., 2010 y Calderón et al., 2014), y por la oleada de once programas de doctorado creados entre 2004 y $2015^{1}$, lo cual supone que las FCA cuentan con capacidades de investigación y de publicación que les permite formar nuevos investigadores.

En la última década (2006-2015) las fuerzas del entorno que inciden en la dinámica de la investigación en las FCA desplazan su énfasis hacia la evaluación de las publicaciones provenientes de su actividad investigativa, y por esta vía cambian los referentes y los parámetros para evaluar el avance de la publicación de las FCA. En la exigencia de incrementar la cantidad y calidad de las publicaciones internacionales confluyen ahora Col-

1 Se crearon en Eafit, Universidades de los Andes, Valle, Rosario, EAN (con dos), del Norte, Medellín, Externado, Simón Bolivar y Jorge Tadeo Lozano (en gestión pública). A ellos se adicionan dos programas de la Universidad Nacional, uno en ingeniería y otro en economía que cuentan con líneas específicas para administración. Además, en 2016 se crearon doctorados en la Universidad Javeriana de Cali y en el ICESI (SNIES, 2016). Así el país cuenta hoy con 15 programas asociados con la administración. 
ciencias, los rankings internacionales -que, como drivers de la globalización, clasifican a las universidades (Shanghai, Qs World University Ranking y su versión latinoamericana QS Latam, THE Times) y a las facultades de administración [Financial Times (MBA), América Economía]-, y las acreditadoras internacionales [AACSB, EFMD (Equis), AMBA]-, a las que han comenzado a acudir en oleada las FCA en busca de una acreditación que mejore su posicionamiento académico y social.

Esa "presión institucional" -como lo denominó Orozco (2015)-, sumada a la competencia tácita entre las facultades, las fuerza a incrementar la cantidad y calidad de sus publicaciones para lograr buenas posiciones en las clasificaciones de Colciencias, los rankings y las acreditadoras internacionales. En términos estratégicos esto significa que se impone un ajuste a los cambios y exigencias del entorno, para mejorar su posicionamiento académico y la legitimidad de su investigación. Cabe esperar, entonces, que estos esfuerzos adaptativos, más allá de las diferencias individuales (Hannan y Freeman, 1977), conduzcan a una mejora más o menos conjunta -isomórfica- de las publicaciones internacionales de las FCA (Dimaggio y Powell, 1983; Whitley, 2008; Orozco, 2015).

El nivel de eficacia de la respuesta de las FCA al nuevo escenario dependerá tanto de contar con condiciones favorables para la investigación, como del desarrollo de recursos y capacidades para investigar, concordantes con las nuevas exigencias del entorno. Pero para dimensionar los cambios requeridos para tales fines es necesario establecer, antes, la magnitud de los desafíos que plantea fortalecer sus publicaciones internacionales -cuestión de la que se ocupa este artículo-.

Ahora bien, en el plano metodológico, para dimensionar los cambios en que se materializa la presión institucional, es preciso dar cuenta de los cambios en los criterios para evaluar la calidad de las publicaciones. De una evaluación centrada en los criterios e indicadores aplicados a las revistas indexadas en el Publindex colombiano, hubo un tránsito progresivo pero acelerado hacia parámetros y estándares internacionales más exigentes. Primero se consideró solo la publicación en revistas indexadas en el Journal Social Science Citation Report (SSCI) de las bases de datos del WoS -de la empresa Thompson Reuters-, reportados en el Journal Citation Report (JCR), y de Scopus -de la empresa Elsevier- reportados en el SCImago Journal Report (SJR); luego se ahondó la exigencia al diferenciar la importancia de los journals en que se publica -por el cuartil ( $Q 1$ a Q4) en que están clasificados-; y finalmente, Colciencias en 2016 extiende las citaciones al "google académico", la aplicación del "índice $h$ " -que representa el más alto número alcanzado entre los $\mathrm{h}$ artículos que han recibido al menos $\mathrm{h}$ citas- para clasificar las revistas académicas 
registradas en el Publindex que no están en WoS o Scopus (Gualdrón, 2016). Los indicios apuntan, además, hacia un uso progresivo de este indicador para evaluar la producción de los investigadores por esa entidad y algunas universidades.

De ese modo, la presión institucional conduce a la aplicación de indicadores de alta exigencia -el cuartil de los journals y la citación- que inducen a publicar en los journals mejor calificados, que son indicadores de alta calidad de la publicación y propician mayor citación. Por lo tanto, la respuesta de las FCA a esa presión se puede establecer por la dinámica de la publicación en los journals indexados en esas bases de datos -WoS y Scopus-. Asunto que se abordará en la siguiente sección. Pero la consolidación de ese proceso genera enormes desafíos y tensiones que es necesario comprender para enfrentarlos adecuadamente. Esto obliga a un doble ejercicio: primero, identificar los contenidos de la discusión internacional en la que se definen los criterios y estándares para la publicación de investigación relevante, pues con ellos y allí es donde se evalúa y se decide sobre la publicación de los artículos producidos en nuestras escuelas; segundo, leer sus implicaciones desde las especificidades y las necesidades de nuestra realidad, para que esa producción tenga impacto no solo académico sino social-empresarial-. Esto se hace en la tercera parte del texto, donde se discuten esos desafíos desde la doble perspectiva señalada y se exploran alternativas para enfrentarlos.

\section{La evolución de la publicación colombiana en los journals internacionales}

En concordancia con los objetivos del trabajo, para establecer la respuesta de las FCA a las exigencias de publicación en journals internacionales de alto impacto, se efectuó una indagación sistemática sobre los artículos publicados en journals tanto del JCR como en el SJR, en el periodo estudiado, y cuyos autores están afiliados a FCA².

La evidencia aportada por las figuras 1 muestra que a mediados de la primera década del nuevo siglo se presenta el punto de inflexión que marcó el despegue de la publicación internacional de los resultados de la investigación sobre la administración en Colombia.

2 Para los artículos primero se identificaron las áreas de conocimiento constitutivas del management; luego se identificaron los artículos en los que uno o más de sus autores estaban vinculados a FCA; después se verificó que los temas cubiertos efectivamente correspondieran al management; luego, en el corpus así conformado se identificaron las revistas en donde se publicaron los artículos y, finalmente, se identificó el cuartil en el que se encontraba la revista el año en que el artículo fue publicado. 
SCOPUS

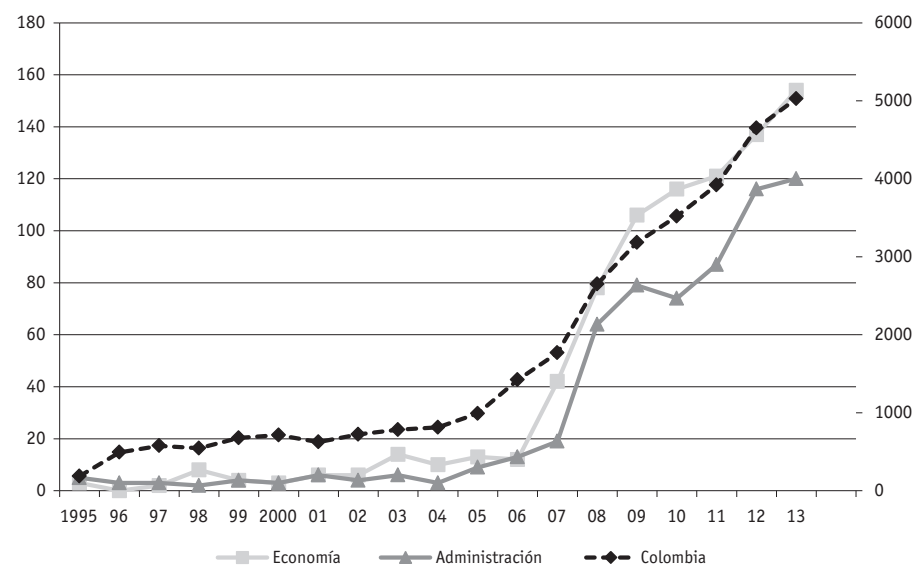

WoS

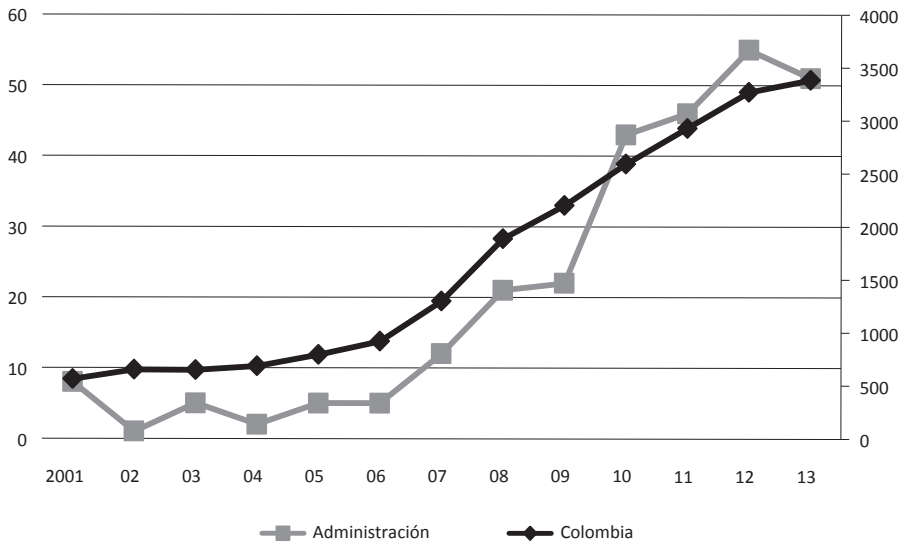

Figuras 1. Publicación de administración de las FCA en WoS y SCOPUS ( $N^{\circ}$ de artículos) Fuente: elaboración propia con información de SCOPUS y WoS.

También muestra que el crecimiento previsto en Malaver (2006) ocurre, pero impulsado ahora por el inicio en 2007 de un vertiginoso incremento del número de publicaciones en journals internacionales admitidos en los dos sistemas de indexación más importantes del mundo Scopus y WoS ${ }^{3}$. Proceso que constituye un nuevo quiebre, el producido por la

3 Cabe advertir que en dicho trabajo la información utilizada era más amplia pues incluía libros, capítulos en libros y artículos en revistas indexadas incluidas Scienti. Aquí se cubre el universo de artículos publicados en Scopus y WoS originado en las FCA entre 2001 y 2014. 
internacionalización de las publicaciones en journals internacionales, que refuerza lo ocurrido a finales de los años 90 (Malaver, 2006).

Una mirada más detenida al comportamiento de la publicación de las FCA revela, por un lado, que esa inflexión hace parte de un proceso más general. Este se presenta al nivel del país en su conjunto, impulsado por la política pública (Malaver y Vargas, 2005), y de él hacen parte las ciencias económicas, con un leve rezago de la administración frente a la economía (figuras 1). Por otro lado, que una rápida comparación con otros países iberoamericanos ilustra (figuras 2) que, con leves diferencias, esa inflexión es simultánea SCOPUS

WoS
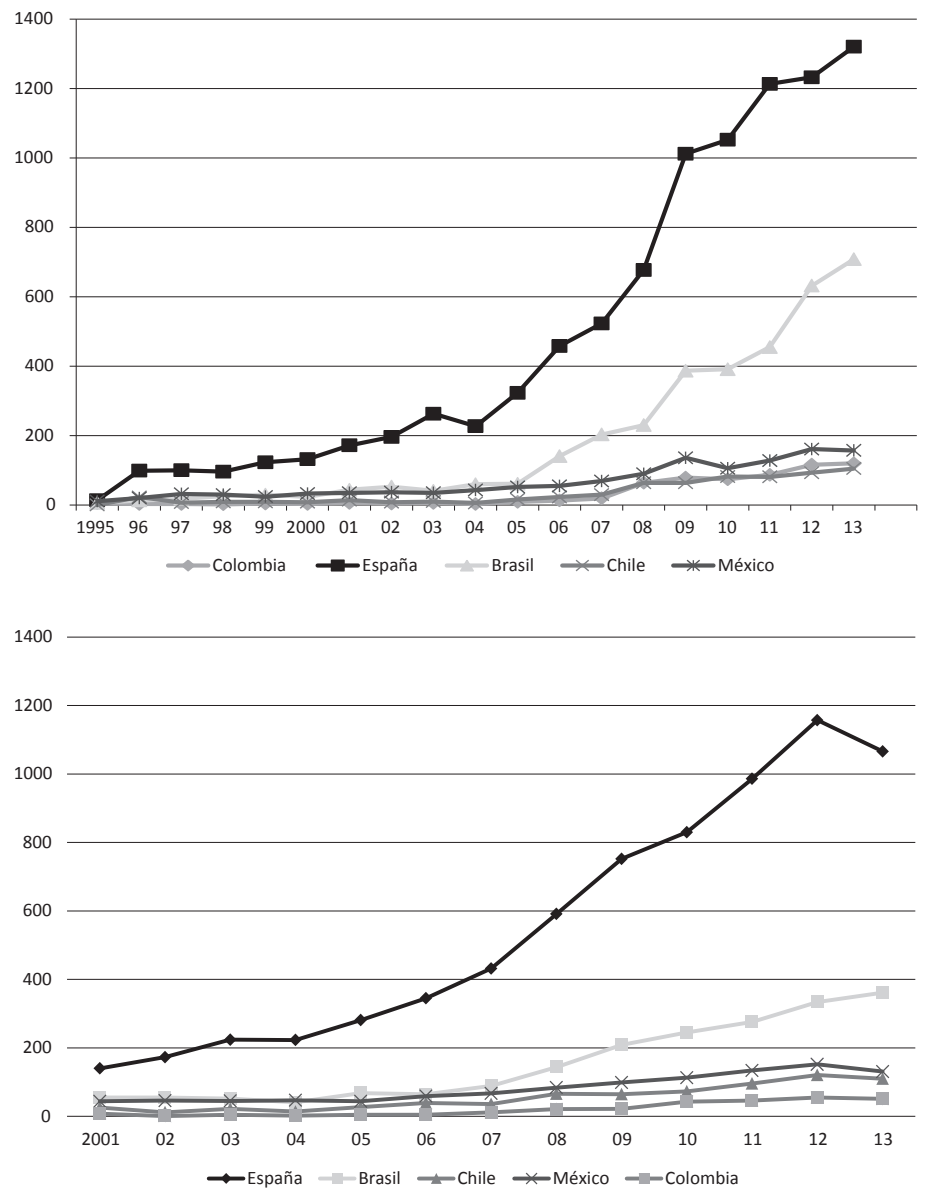

Figuras 2. Evolución de los artículos originados en las facultades iberoamericanas Fuente: elaboración propia con información de SCOPUS y WoS. 
en varios países iberoamericanos, que en España la aceleración inicia en 2005 y es más pronunciada que en los países latinoamericanos, que el proceso es seguido en el tiempo y en el ritmo por Brasil, y que comienza luego en Chile, México y Colombia con ritmo de crecimiento relativamente menor.

El proceso de internacionalización de la publicación de las FCA es simultáneo con el registrado en otros países latinoamericanos. También comparte el bajo punto de partida, expresado en un número de artículos publicados al año que es mínimo en la primera mitad de la década del 2000, como lo indica el promedio anual del total de artículos publicados, que no superaba en Scopus los 3.5 y en la plataforma WoS 2.5 (Tabla 1). Más pequeño se revela al compararse con el número de FCA que superaba las 100 en esos años. Más significativo es que esto aplica incluso a las facultades más reputadas de la región. Así se desprende de Orozco y Villaveces (2015), quienes estudian la producción académica de las 55 facultades con mayor reputación según América Economía y encuentran que entre 2006 y 2011 publicaron 262 artículos en la WoS, es decir, menos de un artículo en promedio al año por facultad, y con mucha diferencias entre ellas.

\section{Tabla 1}

Artículos promedio publicados por las FCA en el periodo 2001-2014 (por cuartil)

\begin{tabular}{cccccc}
\hline Subperiodos & Q1 & Q2 & Q3 & Q4 & Total \\
\hline \multicolumn{5}{c}{ Scopus } \\
2001-2004 & 1,3 & 1,8 & 0,5 & 0,0 & 3,6 \\
$2005-2009$ & 3,0 & 2,8 & 5,4 & 10,0 & 21,2 \\
$2010-2014$ & 15,4 & 8,6 & 25,2 & 12,2 & 61,4 \\
Total (promedio) & 6,7 & 4,4 & 11,0 & 7,9 & 30,1 \\
\hline 2001-2004 & 0,0 & 0,3 & 0,5 & 1,8 & 2,5 \\
$2005-2009$ & 0,4 & 0,2 & 0,4 & 5,6 & 6,6 \\
2010-2014 & 3,2 & 10,2 & 17,2 & 61,8 & 92,4 \\
Total (\%) & 1,3 & 1,3 & 2,2 & 8,6 & 13,4 \\
\hline
\end{tabular}

Fuente: elaboración propia con información de Scopus y WoS.

Esos resultados indican, en síntesis, que es corto el trecho recorrido y mínima la experiencia y aprendizajes en materia de publicación internacional en las FCA y latinoamericanas. De esos resultados puede inferirse, así sea a título de hipótesis, que en términos de la publicación internacional las FCA comparten unos desempeños similares con los 
registrados en otros países latinoamericanos al inicio del proceso, y también ahora en su respuesta a las fuerzas que encarnan la presión institucional y que parece estar operando en esos países. Confirmarlo es importante, pues los aprendizajes que está generando el proceso en esos países podrían aportar luces a las FCA que la pueden ayudar a afinar sus estrategias y acciones para su consolidación.

Ahora bien, al indagar por la evolución de la calidad de las publicaciones de las FCA se encuentra un aumento progresivo en la cantidad de artículos publicados en las revistas de todos los cuartiles. Esto constituye un indicador claro de un aumento progresivo no solo en la cantidad sino también en la calidad -y exigencia- de los artículos publicados en journals internacionales. Los resultados más claros de esa evolución se visualizan en las figuras 3 que facilitan apreciar las características de esa evolución. Si bien existían publicaciones desde comienzos de los 2000, eran aisladas; entonces, como proceso, iniciaron en el segundo quinquenio de esa década, básicamente, a través de la admisión de revistas editadas por FCA tanto en la WoS como en Scopus. Para muchos investigadores estas revistas parecen haberse constituido en el primer paso -y quizá también en el "trampolín"- hacia los journals internacionales publicados en inglés ${ }^{4}$. El crecimiento de los artículos publicados en revistas clasificadas en los cuartiles más altos -01 y Q2- fue más tardío y más tímido, y solo vino a crecer de manera vertiginosa a partir del año 2012. Cabe advertir, que ahondar para comprender los aprendizajes implícitos en ese proceso sería muy útil para efectos de la formación ofrecida en los programas de doctorado.

Las cifras de esa evolución son tanto o más elocuentes: muestran que en el primer lustro de los 2000 la publicación era tan escaza que constituía un evento aislado, y era prácticamente inexistente la publicación de artículos en revistas Q1 (Tabla 1); muestran un crecimiento exponencial sobre todo en las revistas de mayor cuartil (Q1 y Q3). El peso de esa expansión es tan importante, que en el último lustro se publicaron cerca de las tres cuartas partes del total de las publicaciones efectuadas entre 2001 y 2014, y es mayor en las revistas 01 , donde ese porcentaje supera el $80 \%$, y en los dos últimos años bordea el $50 \%$ del total (Tabla 2). Por lo tanto, allí la progresión al final del periodo es mayor y cabe esperar que a futuro lo sea aún más. Esto, debido a los aprendizajes que se vislumbran

4 La importancia de estas revistas se revela crucial en este periodo. Profundizar en su papel a futuro es crucial, sobre todo, de cara a la discusión que se adelanta en la sección 3; pero el análisis específico de estas revistas amerita un trabajo específico. Aquí baste señalar que su comportamiento fue bastante inestable, y esto estuvo asociado en el caso de Scopus a la Revista Cuadernos de Administración, que estuvo clasificada en Q3 entre 2009 y 2013 y luego descendió a Q4; y en el caso de la WoS, debido a Innovar, que estuvo indexada en el SSCI en 04 entre 2010 y 2012 y luego aparece desindexada. 
SCOPUS

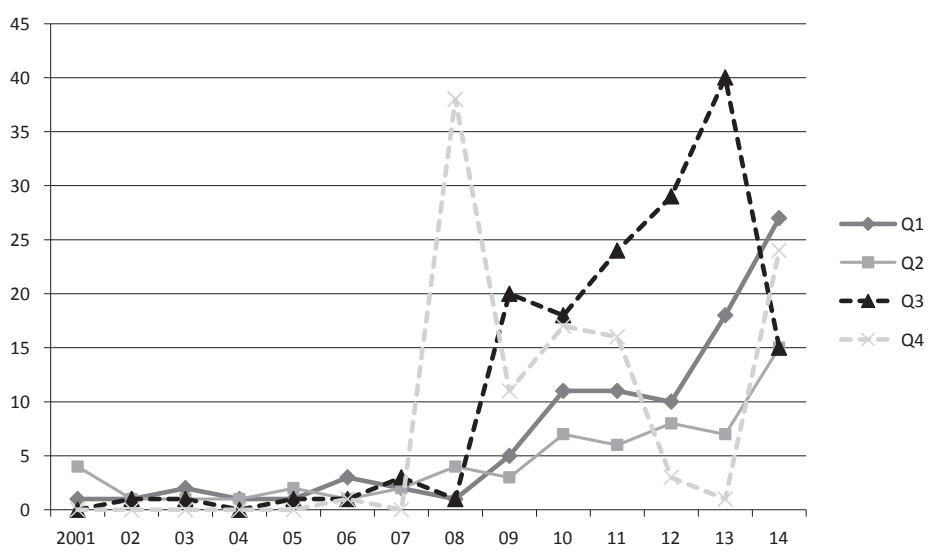

WoS

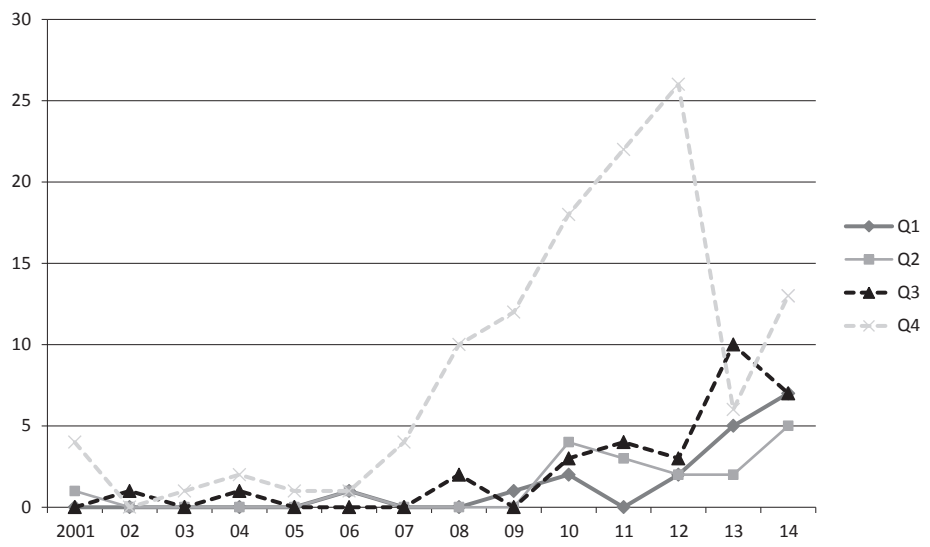

Figuras 3. Evolución de los artículos originados en FCA por cuartiles

Fuente: elaboración propia con información de SCOPUS y WoS.

al final del periodo de análisis y a que pareciera que publicar en revistas $Q 1$ se ha convertido en un objetivo casi que existencial para los investigadores, en el nuevo contexto de evaluación de su desempeño, ligado a la calidad de sus publicaciones (asunto sobre el cual se volverá más adelante).

La indagación por el destino -geográfico- de los artículos publicados por investigadores vinculados a las FCA muestra un vertiginoso crecimiento entre 2010 y 2014 en las tres regiones consideradas, siendo mayor en Europa que en Norteamérica y Latinoamérica (Tabla 3). Pero en todos los casos, ese crecimiento es tan alto en este quinquenio que supera el $70 \%$ 
de los publicados en todo el periodo analizado. Ese aumento, fue tan pronunciado que de un promedio anual de artículos publicados en conjunto en Europa y Norteamerica, de 2.5 en la WoS y y 3.5 en Scopus, ese promedio creció hasta 14 y 30 en el último subperiodo. Esto muestra la fuerza del proceso de internacionalización, pero a una escala muy pequeña todavía para una comunidad que cuenta con 181 instituciones que ofrecen programas de pregrado y posgrado en administración (SNIES, 2016); y lo es incluso para el número de facultades que han empezado a ofrecer programas de doctorado, pues a duras penas publicarían el promedio de dos (2) artículos en Scopus y uno (1) en la WoS.

Tabla 2

Evolución de las publicaciones por cuartiles y subperiodos, 2001-2014

\begin{tabular}{cccccc}
\hline Subperiodos & Q1 & Q2 & Q3 & Q4 & Total (\%) \\
\hline \multicolumn{5}{c}{ Scopus } \\
\hline 2001-2004 & 5,3 & 11,3 & 1,3 & 0,0 & 3,3 \\
$2005-2009$ & 12,8 & 19,4 & 16,9 & 45,0 & 23,8 \\
$2010-2014$ & 81,9 & 69,4 & 81,8 & 55,0 & 72,9 \\
\hline Total (\%) & 100,0 & 100,0 & 100,0 & 100,0 & 100,0 \\
\hline \multicolumn{7}{c}{ WoS } \\
\hline 2001-2004 & 0,0 & 5,6 & 6,5 & 5,8 & 5,3 \\
$2005-2009$ & 11,1 & 5,6 & 6,5 & 23,3 & 17,6 \\
$2010-2014$ & 88,9 & 88,9 & 87,1 & 70,8 & 77,0 \\
\hline Total (\%) & 100,0 & 100,0 & 100,0 & 100,0 & 100,0 \\
\hline
\end{tabular}

Fuente: elaboración propia con información de SCOPUS y WoS.

\section{Tabla 3}

Evolución de las publicaciones de las FCA, por regiones y subperiodos

\begin{tabular}{ccccc}
\hline Periodo & América Latina & Europa & Norte América & $\mathrm{N}^{\circ}$ artículos \\
\hline $2001-2004$ & 0.0 & WoS & & \\
$2005-2009$ & 27.2 & 7.8 & 11.9 & 10 \\
$2010-2014$ & 72.8 & 81.3 & 9.5 & 33 \\
Total (\%) & 100 & 100 & 18.6 & 144 \\
\hline $\mathrm{N}^{\circ}$ total artículos & 81 & 64 & 42 & 187 \\
\hline & & Scopus & & 14 \\
\hline $2001-2004$ & 0.0 & 5.6 & 8.0 & 100 \\
$2005-2009$ & 28.1 & 19.4 & 19.5 & Continúa
\end{tabular}




\begin{tabular}{ccccc}
\hline Periodo & América Latina & Europa & Norte América & $\mathrm{N}^{\circ}$ artículos \\
\hline $2010-2014$ & 71.9 & 75.0 & 72.4 & 307 \\
\hline Total $(\%)$ & 100 & 100 & 100 & 421 \\
\hline $\mathrm{N}^{\circ}$ total artículos & 210 & 124 & 87 & \\
\hline
\end{tabular}

En WoS, en Europa se incluye un artículo publicado en Australia.

En Scopus, en Europa se incluye un artículo publicado en Singapur.

Fuente: elaboración propia con información de SCOPUS y WoS.

La composición geográfica de las publicaciones internacionales revela varios hechos significativos: primero, que cuando las revistas colombianas ingresan a Scopus y la WoS en el segundo lustro de la década de 2000, tienen una importancia fundamental para la internacionalización, no solo por el alto porcentaje que representan (Tabla 4), sino por empezar a exigir los estándares de esos sistemas de indexación; segundo que luego los investigadores se orientan a la publicación en journals europeos y norteamericanos, a tal ritmo que en el último quinquenio superaron la cantidad de artículos publicados en revistas latinoamericanas, las cuales después de servir de trampolín, no tienen un futuro claro en el proceso de internacionalización; tercero, que cuando se comparan las revistas europeas y norteamericanas, las europeas representan en ambas bases de datos cerca del $60 \%$ de los artículos publicados, quizá por el peso de las revistas españolas. Finalmente, en el Anexo 1 se presentan las 20 revistas con mayor número de publicaciones (entre 2001 y 2014) de investigadores vinculados a las FCA.

\section{Tabla 4}

Composición de las publicaciones de las FCA, por regiones y subperiodos

\begin{tabular}{ccccc}
\hline Periodo & América Latina & Europa & Norte América & Total (\%) \\
\hline $2001-2004$ & 0.0 & WoS & & \\
$2005-2009$ & 66.7 & 50.0 & 50.0 & 100 \\
$2010-2014$ & 41.0 & 36.1 & 12.1 & 100 \\
\hline $2001-2014$ & 81 & 64 & 22.9 & 100 \\
\hline $2001-2004$ & 0.0 & Scopus & 42 & 187 \\
$2005-2009$ & 59.0 & 50.0 & 50.0 & 100 \\
$2010-2014$ & 49.2 & 24.0 & 17.0 & 100 \\
\hline $2001-2014$ & 210 & 30.3 & 20.5 & 100 \\
\hline
\end{tabular}

En WoS, en Europa se incluye un artículo publicado en Australia.

En Scopus, en Europa se incluye un artículo publicado en Singapur.

Fuente: elaboración propia con información de SCOPUS y WoS. 
En síntesis, dado lo reciente del proceso de internacionalización de la publicación en las FCA y la escasa producción que se publica en revistas europeas y norteamericanas, es decir, la poca experiencia y aprendizajes acumulados sobre las lógicas y dinámicas de los medios -journals- de circulación del nuevo conocimiento disciplinar, puede afirmarse que el proceso de internacionalización de las publicaciones todavía está en ciernes y que, en consecuencia, son grandes los desafíos que plantea ampliar y consolidar la publicación en los journals internacionales de mayor nivel de calidad. Enseguida se ahondará en el análisis de algunos de esos desafíos y de las tensiones que llevan aparejadas.

\section{La publicación internacional de las FCA: tensiones, desafíos y relevancia}

Para dimensionar el alcance de los desafíos que implica aumentar la publicación internacional de calidad el punto de partida, hoy, es comprender el significado de la discusión actual sobre la relevancia de la investigación publicada en journals de administración. Este debate trasciende la dimensión académica y se extiende a la utilidad de la publicación para la sociedad y las organizaciones. Se expresa, en síntesis, en la crítica al privilegio del rigor sobre la relevancia, que trasciende el ámbito de la investigación y se extiende a las escuelas de negocios. Se señala que la adopción -en la segunda mitad del siglo XXdel "modelo de excelencia académica" centrado en la investigación científica hecha a la manera de las ciencias naturales y de modelos matemáticos "reduccionistas" condujo a privilegiar el rigor-metodológico-sobre la relevancia -práctica- (Pfeffer y Fong, 2002; Mintzberg, 2005; Bennis y 0'Toole, 2005). Por esta vía las escuelas ganaron rigurosidad científica, pero a costa de su crisis de legitimidad en el ámbito de los negocios (Thomas y Wilson, 2011).

Esto ha generado reacciones en el ámbito de la investigación orientadas a reducir los sesgos del modelo de ciencia imperante a favor de mayor equilibrio entre las vías deductivas e inductivas, los métodos cuantitativos y cualitativos, el rigor y la relevancia. Se pide evaluar la publicación por su originalidad -su contribución a la teoría- y su utilidad -para explicar y solucionar problemas empresariales y sociales- (Corley y Gioia, 2011); también desarrollar formas de investigación que permitan tender puentes entre académicos y practitioners (Bartunek y Rynes, 2014). En síntesis, ese debate amplía los espacios para la publicación internacional, y permite vislumbrar los desafíos -y las tensiones que les son inherentes- para las FCA. Aquí nos concentraremos, en particular, en la tensión entre el rigor y la relevancia, la derivada de aproximaciones centradas en la 
relevancia, y la de convertir al contexto en fuente de nuevo conocimiento; todas ellas de cara al desafío de la publicación.

\subsection{El desafío de la relevancia y la tensión entre rigor e imaginación}

Un artículo con el perfil de "publicable", debe contribuir al avance del conocimiento. Irónicamente, hoy se critica que frente al elevado crecimiento del número de artículos publicados en los journals de administración es poca la originalidad del "nuevo" conocimiento generado (Alvesson y Sandberg, 2013). Esto se debe a la lógica de la publicación ${ }^{5}$. Esta se basa, por un lado, en revisiones exhaustivas orientadas a mostrar el conocimiento de la literatura y a identificar un vacío de conocimiento - generalmente puntual- a cubrir, que constituye la contribución del artículo; por otro, en la aplicación de un sofisticado aparataje metodológico como indicador de rigor, condición sine qua non para su publicación. La verificación del cumplimiento de esos criterios corre a cargo de los editores y evaluadores de los journals. Esto lleva a contribuciones puntuales, poco originales e imaginativas, pero que extienden el paradigma dominante -en el sentido de Kuhn (1962)que articula la comunidad académica que tiene en los editores y evaluadores de dichos journals a los guardianes de su preservación y desarrollo.

Allí hay poco espacio para problemas emergentes, complejos, desafiantes, cuya solución implique cuestionar los paradigmas, desafiar sus supuestos teóricos y heurísticas de indagación; para explorar en disciplinas afines en búsqueda de planteamientos, lenguajes y metodologías alternativas a las existentes o que escapen al dominio de conocimiento y experticia de los revisores de los journals (Alvesson y Sandberg, 2013; George, 2014). Estos cuestionamientos encuentran sustento empírico en Rafols et al. (2013) quienes muestran que en los journals top en administración predominan los trabajos monodisciplinares, pese al carácter interdisciplinario del management.

Kuhn (1962) ofrece valiosos elementos para enfrentar los desafíos originados en esa situación cuando plantea que los mayores avances en el conocimiento surgen de problemas que no se pueden solucionar con las explicaciones y metodologías de los paradig-

5 Impulsada por tres drivers: las políticas institucionales de las facultades, que dan prioridad a la publicación en journals de alto impacto para efectos de la promoción y permanencia de sus profesores; la constitución de la identidad del investigador, con base en los journals donde publican -asemejada al “dime dónde publicas y te diré quién eres"-; y las normas profesionales para la publicación académica, materializadas en los formatos y estándares de los journals, regentados por sus editores y revisores (Alvesson y Sandberg, 2013). 
mas dominantes, esto es, la ciencia normal, sino con la ciencia "anormal" -paradigmas emergentes-. Pero esto implica rupturas, desafiar el consenso en que se apoya el paradigma dominante. También se pueden encontrar alternativas en las voces que al interior del management piden privilegiar el rigor intelectual enfocado en la pregunta -en la problematización de la teoría, en la identificación de problemas y de material empírico que desafíe sus supuestos-; es decir, en la exigencia e imaginación teórica antes que el énfasis en la sofisticación metodológica (Mintzberg, 2005; Alvesson y Sandberg, 2013); sobre todo, cuando esta pareciera reducir el rigor al ámbito de las metodologías cuantitativas (Donaldson et al., 2013). Rigor que, por lo demás, facilita afinamientos y avances incrementales, especialmente, en paradigmas maduros.

En esa dirección también se están moviendo equipos editoriales de journals top, cuando afirman que han adicionado -a la contribución teórica y empírica; a lo interesante y novedoso; a la claridad expositiva- un nuevo criterio para evaluar los artículos: si "aborda un problema importante para las organizaciones", y que además prefieren "manuscritos que enfaticen en cómo los constructos proveen una explicación coherente de un fenómeno antes que enmarcar y motivar estudios por adicionar moderadores y mediadores no probados" (George, 2014, p. 2). Es decir, asumen la crítica a la irrelevancia de muchos de los artículos publicados. De ese modo, se amplían los espacios para la investigación preocupada por la relevancia ${ }^{6}$.

\subsection{La relevancia, los modos de producción de conocimiento y la publicación}

Mirado el debate del rigor y la relevancia desde una perspectiva más general, puede decirse que el modelo de investigación asumido por las escuelas de negocios se asemeja al "modo uno" de investigación, a un "modelo de oferta" que se tradujo en la problemática expuesta en la sección anterior. Una alternativa es el llamado "modo dos" de producción de conocimiento, que responde al interés de las universidades por contribuir a solucionar problemas de la sociedad, por ser relevantes para el contexto en que actúan (Gibbons et

$6 \quad$ Cabe advertir que no se desconocen las fortalezas del modelo imperante ni sus avances, que son significativos, por ejemplo, en los análisis longitudinales con datos panel, o en los estudios experimentales, que profundizan sus propósitos explicativos. Tampoco se desconoce la importancia de la investigación básica que bastante falta hace en nuestro contexto, como se presentará más adelante. Se pretende, sí, mostrar que se están abriendo espacios para otras maneras de hacer ciencia, y que ellos amplían las avenidas y posibilidades de publicación internacional. 
al., 1994); y por ello construyen sus problemas y agendas de investigación desde y con la sociedad ${ }^{7}$.

Transfield y Starkey (1998) mostraron su pertinencia para construir conocimiento en su contexto de aplicación, para resolver problemas que por su naturaleza son de carácter interdisciplinario entre académicos y practitioners. Esta constituye una vía para tender puentes entre estos dos actores y aumentar la relevancia. Pero tienen diferencias en sus intereses, tiempos, formas de conocer y de comunicarse, etc., de las que se derivan fuertes tensiones, insuperables, y que requieren un manejo creativo de las mismas (Bartunek y Rynes, 2014). La publicabilidad de los resultados de ese modo de investigar plantea desafíos y tensiones, que son extendibles a la investigación "orientada por problemas" prácticos, abordados de manera inductiva y con métodos cualitativos, en los que es necesario detenernos.

De entrada, para definir la magnitud de los desafíos, es vital dimensionar el alcance y novedad del problema estudiado. Si el problema es puntual o local, y para solucionarlo son suficientes los conocimientos y metodologías existentes, el estudio se asemeja a una consultoría y difícilmente dará lugar a publicaciones en revistas de investigación. Si tanto el problema como su solución son aplicables e interesantes en otros contextos, es clara su relevancia empírica y es útil e incluso necesaria su publicación, pero en revistas enfocadas en practitioners.

Cuando se requieren ajustes, adaptaciones o desarrollos, para que el conocimiento existente funcione en ese contexto (o contextos) de aplicación, se debe validar y extender la teoría - a la manera de Yin (2003)-. En este caso existe una contribución al conocimiento que posibilita su publicación en revistas académicas. Esas publicaciones serán fruto de un diálogo doble: con actores empresariales -en su origen y en la aplicación de sus resultados- y con las comunidades académicas de referencia -que validan y aprueban su publicación-.

7 Aquí son de extrema utilidad los avances en las políticas de estímulo del vínculo universidad - empresa - estado, y los planteamientos analíticos que las inspiran, por ejemplo, los de Etzkowitz y Leydesdorff (2000), Etzkowitz et al. (2000), Leydesdorff (2012). Ahondar al respecto escapa a los objetivos de este trabajo; no obstante, es necesario señalar que aquí también existe la tensión entre, por un lado, estímulos y dinámicas de las alianzas que pueden circunscribirse a los ámbitos regionales o nacionales y, por otro, mercados y ciencias impulsadas por dinámicas más globales. En otros términos, entre resultados de investigaciones que resuelven problemas locales y conocimientos generados por éstas que deben ser atractivas -publicables-al nivel internacional. 
Si una indagación rigurosa confirma que la teoría existente no puede explicar el problema identificado, es preciso emprender el largo viaje de construcción del conocimiento requerido para solucionarlo. Proceso que -sin ahondar aquí- implica ejercicios exploratorios para identificar los rasgos que caracterizan el problema, los que se repiten y posibilitan establecer patrones o los hechos estilizados (Helfat, 2007); los que permiten elaborar interpretaciones a contrastar con las teorías existentes en un proceso iterativo -abductivo- que lleva, finalmente, a la emergencia del nuevo conocimiento -constructos y conceptos- generado durante este proceso.

La complejidad de ese proceso es alta y, según sus orientaciones, el investigador puede acudir a marcos alternativos como los estudios de caso a profundidad (Eisenhardt y Graebner, 2007) o la teoría apreciativa (Nelson, 1995). Pero es probable que en no pocos estudios, el problema (la realidad) estudiado sea mucho más complejo, y que el proceso sea mucho más arduo, largo y dispendioso, que el planteado por Eisenhardt $(1989,1991)$ para los casos, y que se requiera más de un estudio para abordarlo y que sus resultados no puedan ser captados y publicables en un solo artículo.

Es probable que incluso en aquellos casos en que la novedad del conocimiento generado durante ese proceso sea indudable, estará latente la tensión de enfrentarse en el ámbito de la publicación con pares devotos de la lógica deductiva de la ciencia y de los paradigmas dominantes, es decir, que el “modo dos" sea juzgado por el “modo uno" (Bartunek, 2011) y, en consecuencia, apliquen la plantilla -estándar- de la evaluación típica: el vacío y la contribución -puntual-, las hipótesis, etc. Allí, la probabilidad de caer en la problemática definida en la sección anterior es alta, sobre todo para los artículos originados en las etapas iniciales -exploratorias- del proceso, donde las aproximaciones al problema son inherentemente inductivas y cualitativas. Por supuesto existe el riesgo opuesto: que en esos procesos no haya ni rigor ni relevancia. Al respecto, cabe aclarar, que no hay relevancia sin rigor. El problema es de orden, pues el rigor no garantiza la relevancia.

\subsection{El contexto y la relevancia}

La consideración explícita del contexto, más precisamente de las diferencias contextuales como fuente de conocimientos emergentes, está ampliando los espacios para generación de conocimiento y su publicación. De hecho, sociedades con diferencias en sus culturas e instituciones, en sus valores y visiones del mundo, y en sus niveles de desarrollo, tienen problemáticas y prácticas gerenciales distintas (Barkema et al., 2015). Diferencias de las que, por definición, debería dar cuenta la investigación publicada; sobre todo en el 
caso management, que "trabaja en el margen entre la ciencia social básica y la aplicada" (Barley et al., 1988, p. 24).

Eso nos remite a la cuestión de quién plantea la pregunta que orienta la investigación de punta, para resolver qué problemas, de qué realidad. En el management, los investigadores de los países desarrollados que publican en journals -editados en inglés-generan un diálogo académico -sobre problemáticas asociadas con su realidad organizacional y gerencial-, fruto del cual emergen los nuevos conocimientos y los consensos que configuran los paradigmas dominantes. Publicar allí significa intervenir directamente en esa conversación, dinamizándola. Aunque deseable, esta alternativa es difícil para investigadores de otros contextos, porque su aproximación a esa realidad está y estará mediada en la mayor parte de los casos. Si el investigador supera las distancias y mediaciones y hace aportes teóricos, aun así, dado el carácter contextual de las ciencias sociales, surgirá la pregunta por la aplicabilidad de su contribución a su realidad, esto es, por la pertinencia de su investigación.

Una vía para superar esa tensión es asumir los contextos específicos pues, al hacerlo, la rica variedad de problemáticas que emerge amplía las posibilidades analíticas. Si, en nuestro caso, las realidades y problemáticas de los países en desarrollo difieren de las existentes en los países desarrollados, entonces los marcos analíticos deberán ampliarse, adaptarse o crearse para explicarlas. Pero a condición de contrastarlos, de dialogar con ellos, desarrollando conocimiento propio, que enriquece y amplía el existente.

Esa vía se ha mostrado plausible para los países asiáticos. Ayer, debido a sus "milagros económicos" y al éxito de sus empresas, de sus prácticas gerenciales y formas organizacionales particulares -como la gerencia japonesa, los kereitzu y los chaeboles; 0 empresas específicas, como Toyota o Hyundai-, que despertaron el interés por el éxito y contundencia de sus ventajas competitivas, y suscitaron una oleada de publicaciones orientadas a explicarlos y a derivar lecciones para la política pública y el management (Nelson y Pack, 1999; Banco Mundial, 1993; Coriat, 1992; Kim, 1998). Hoy, debido al boom económico de China o el desplazamiento de la producción industrial del conjunto de países de la OECD por los países asiáticos, que despierta igual interés por las “lecciones" que de allí se pueden derivar.

Pero en conjunto, la contribución a la teoría no ha sido fácil. En su diagnóstico sobre la internacionalización académica del management asiático, Barkema et al. (2015) señalan que ésta ha sido poco exitosa, porque el $18 \%$ de los miembros del Academy of Manage- 
ment son asiáticos y solo han contribuido con el $8 \%$ de los artículos publicados en el Academy of Management Journal entre 2010 y 2014; porque hay un fuerte crecimiento en estudios basados en datos asiáticos pero estos aplican teorías -y miran su realidad con lentes- occidentales y, en consecuencia, aportan muy pocos conceptos y teorías nuevas. Esto a pesar de las grandes diferencias en su cultura, filosofía, valores e instituciones y sus manifestaciones en las prácticas administrativas. Por ello, estos autores invitan a investigar sobre nuevos y/o relevantes problemas económicos y sociales de sus propias realidades, antes que llenar vacíos en los paradigmas existentes, esto es, trabajar en el vecindario de lo conocido e incremental de la "ciencia normal".

Para nuestro caso, podría aducirse que problemas latinoamericanos persistentes -como el rezago tecnológico y en las prácticas gerenciales- parecerán anacrónicos y de poco interés para las revistas top, no obstante, el problema principal parece radicar en la actitud frente al conocimiento y la realidad propia. De hecho, las evidencias indican que en América Latina ha predominado la copia y replica sin adaptación, de las teorías y modelos anglosajones (Fernández y Gantman, 2011). Y esa dependencia -colonialismo epistemológico lo denomina Ibarra (2006)- hoy resulta tan disfuncional, como su contraparte: la pretensión del universalismo científico -imperialismo americano para Augier, March y Sullivan (2005)-, que es una muestra del parroquialismo norteamericano, ante los desarrollos previsibles en Europa y Asia (Augier et al., 2005). De ese modo, la construcción de un pensamiento inédito sobre nuestra realidad podría contribuir a ampliar el diálogo con otras comunidades académicas, a partir del conocimiento originado en la diversidad contextual.

\subsection{Un corolario: la necesidad de indicadores de relevancia}

Un cambio significativo de la presión institucional orientada a la mejora de la publicación de las FCA, como se dijo, es la evaluación por la citación, como indicador generalizado del impacto de la publicación científica derivada de la investigación. Esta representa un avance al moderar la tendencia a convertir un medio -la publicación- en un fin, y la exacerbación del vacío puntual proclive al incremento en la cantidad de publicaciones bien hechas pero irrelevantes. Sin embargo, también es fuente de tensiones y desafíos inevitables y que, por lo tanto, es necesario considerar.

La citación genera el "efecto Mateo" (Merton, 1968), expresado en que "quien tiene más citas recibirá más citas", debido a que produce una especie de "conexión preferencial" que se traduce en que, ante un descubrimiento simultáneo, recibe más citas quien 
es más conocido. De ese modo se pervierte el principio de que no existe autoridad en la ciencia (AACSB, 2011), y se ahoga a los autores -y revistas- más jóvenes y con pocas citaciones. En la misma dirección, cabe esperar -como conjetura- que "la inmediatez" y las citaciones afectarán negativamente a las contribuciones originales y relevantes pero al margen del mainstream; también a las que son fruto de investigación -aplicada- más cercana a la práctica profesional.

La citación también conlleva distorsiones derivadas de la historia de su aplicación, ligada a dos empresas privadas con ánimo de lucro -Thomson Reuters y Elsevier, que conformaron una especie de duopolio en el espacio de la publicación de artículos científicos-. Estas aplican y divulgan a través del JCR y del SJR las evaluaciones de los journals admitidos e incorporados en sus bases de datos; evaluaciones que están basadas en el "factor de impacto" o el "índice h", subordinados a su vez a la citación. Al estar supeditadas dichas evaluaciones a la información contenida en las bases de datos de estas dos empresas y al estar condicionado el acceso -a dichas evaluaciones y a los artículos- al pago a ellas, se atenta contra el principio del carácter abierto del acceso al conocimiento científico. Con el agravante de que tales evaluaciones se han institucionalizado y se han convertido en norma que aplican gobiernos, entidades financiadoras, universidades y las mismas FCA para evaluar la calidad de las publicaciones.

En la evolución de las publicaciones internacionales originadas en las FCA, que aquí nos ocupa, se pueden ver el peso y los sesgos introducidos por esos dos sistemas de indexación. Estos se visualizan en el momento del despegue de la publicación internacional, en el segundo lustro de la década de 2000, cuando varias revistas editadas en FCA -Academia, Innovar, Cuadernos de Administración- fueron admitidas en WoS y Scopus, pues es solo a partir de este momento que sus artículos comenzaron a ser contabilizados y evaluados en los dos sistemas de indexación (tablas 2 y 3 ). Los artículos publicados con anterioridad fueron "invisibilizados" independientemente de las citaciones posteriores recibidas, ya que solo se contabilizan los artículos que hacen parte de los fascículos publicados a partir de la fecha de indexación de las revistas. Pero para ese momento, esas revistas ya estaban internacionalizadas por los autores de los artículos, los evaluadores y los integrantes de sus comités editoriales; por su ámbito de circulación, como por los directorios académicos -como Ulrich, Scielo, Latindex, RedAlyc-y comerciales -como JSTOR o Ebsco- en que estaban admitidas.

Por ello, movimientos como los del Open Acces Journals representan un avance en la democratización del acceso al conocimiento científico publicado en journals. También, 
la evaluación de las publicaciones -journals, artículos y autores- hecha por Google a través del "índice $h$ ", divulgado gratuitamente en el Google académico, pues capta las citaciones recibidas por diferentes tipos de publicaciones -libros y capítulos de libros, artículos, etc.- durante la vida académica de los investigadores. Esto aumenta la visibilidad de la producción académica de los investigadores, pero su debilidad proviene de los criterios de calidad aplicados para su inclusión en esa base de datos. De allí la necesidad de indicadores complementarios para una adecuada valoración de la calidad de la producción académica publicada.

Sin embargo, el principal problema de la citación es su alcance parcial, pues está orientada a evaluar la influencia académica del artículo, no su impacto y relevancia empresarial o social. Y la falta de un indicador específico para su utilidad ${ }^{8}$ afecta especialmente la valoración de la investigación aplicada, que es fundamental en un campo tan ligado a la práctica profesional, que -a semejanza de la medicina- es fuente de nuevo conocimiento, y que puede encontrar en el "modo dos" de investigación una vía para su desarrollo. Esta carencia restringe la publicación, la visibilidad y la legitimidad del conocimiento producido en las FCA.

La falta de criterios indicadores para evaluar, valorar y reconocer la contribución de la investigación a mejorar las organizaciones y su sociedad, desincentiva la investigación aplicada, amplía la brecha entre la universidad y la empresa, y deja un espacio vacío que es copado por una literatura producida -generalmente- por consultores y gurús, que suele convertirse en "modas administrativas" cuya lógica y efectos fueron analizados con agudeza, en nuestro medio, por López (1998). Este autor muestra cómo sin el sustento de investigación previa, sin especificar sus alcances organizacionales o las condiciones para su aplicación, y sin evaluación alguna de los efectos de su aplicación, estas publicaciones venden soluciones que, con tono mesiánico y carácter prescriptivo, "garantizan el éxito empresarial". Se apoyan en frases rimbombantes y en un anecdotario de experiencias de personas o de empresas exitosas en su aplicación; en comentarios de personajes citados con criterio de autoridad; o en el éxito en ventas de un texto novedoso convertido en best seller. Suelen aplicarse -viralmente- de manera acrítica y dogmática, como recetas sencillas, novedosas, iluminantes e inspiradoras; sin diagnóstico de las necesidades o de

8 Y las alternativas como el ALTmetrics, no representan una solución siquiera parcial a esta situación. Algunos ejemplos de métricas e indicadores -como la adopción de nuevas prácticas derivadas de la investigación, 0 de nuevas y/o mejores metodologías para desarrollar actividades organizacionales- pueden encontrarse en AACSB (2012). 
las adecuaciones requeridas en la organización para aplicarlas, o de las adaptaciones de dicha "teoría" para su aplicación en ese contexto organizacional. Los esfuerzos y recursos invertidos en su implantación suelen ser infructuosos; pero esto recibe poca atención pues el énfasis está puesto en el nuevo best seller o "teoría" -moda- administrativa.

Superar la carencia de indicadores que revelen, visibilicen y contribuyan a mejorar la valoración de la utilidad social de la investigación en que se basa la publicación ayudará, entonces, a enfrentar tensiones que trascienden el ámbito de la publicación. Contar con ellos estimulará la producción de conocimiento en las FCA que contribuirá a cumplir con la responsabilidad de entender las causas y mecanismos a través de los cuales operan y se reproducen los problemas que enfrentan las organizaciones; a identificar, analizar y -por qué no- a desarrollar y a divulgar prácticas innovadoras replicables y, a superar de esa manera, la fractura universidad - empresa. Pero en las circunstancias actuales esto implica ir más allá de la evaluación por la citación que impone la presión institucional.

\section{A manera de balance}

La indagación efectuada confirma la tendencia creciente de las publicaciones previsto por Malaver (2006), en razón de la política pública de desarrollo de capacidades para investigar y divulgar el nuevo conocimiento producido, vía grupos de investigación y revistas académicas, y de normas que contribuyeron a la institucionalización de la actividad investigativa en las FCA. Muestra además, que en respuesta a "la presión institucional" -ejercida por la política pública, los rankings y acreditadoras internacionales-, se produjo desde la segunda mitad de la década de los 2000 un segundo despegue de las publicaciones, esta vez, en journals internacionales de alta calidad. Y con ello se refuerza el quiebre histórico, que en materia de investigación y de la publicación de sus resultados se produjo entre la segunda mitad de años 90 y de los 2000, frente a la evolución registrada en las tres décadas anteriores.

La evidencia analizada sugiere que las fuerzas que dan vida a la presión institucional están empujando el proceso de internacionalización de las publicaciones de manera transversal en las distintas disciplinas científicas colombianas, así como en el campo del management en Iberoamérica. Si a esto se suman los aprendizajes generados por ese proceso y por la oferta de educación doctoral ocurridos durante los últimos diez años, es plausible esperar un aumento en la cantidad y la calidad de las publicaciones de las FCA. Pero el bajo nivel del que se parte y el poco trecho recorrido indica que el proceso está apenas en ciernes y, esto, la acelerada adopción de cada vez más exigentes parámetros 
y estándares internacionales para evaluar la producción académica, genera desafíos y tensiones descomunales para las FCA en el corto plazo.

El análisis de algunos de esos desafíos y tensiones muestra que además de la originalidad hoy se valora la relevancia del conocimiento producido. La discusión internacional sobre la relevancia abre espacios (incluso en journals top) para ir más allá del criterio rector convencional -la contribución centrada en llenar el gap identificado en la teoría-, y valorar el abordaje y solución de un problema empresarial/social relevantes, como fuente de nuevo conocimiento; y por esta vía también se abre espacio a la geografía, esto es, a la diversidad de culturas, visiones de mundo y prácticas empresariales que le son concomitantes como fuente potencial de nuevos conceptos y teorías. Por ello, las particularidades del contexto institucional, culturaly de las prácticas administrativas de las organizaciones latinoamericanas, son reservorio de oportunidades para enriquecer el conocimiento existente, y legitimar su publicación. Esto supone, desde luego, asumir y valorar la realidad propia, como está sucediendo en otros contextos.

El análisis también muestra que la citación como base para definir el impacto, y como indicador de la influencia en investigaciones posteriores y el cuartil de los journals en que se publica, conlleva sesgos, es limitado para captar la contribución al desarrollo empresarial y la utilidad social del conocimiento divulgado. Esto afecta a la investigación aplicada, el reconocimiento y legitimidad social y empresarial de las FCA. De allí la urgencia de diseñar indicadores complementarios que posibiliten una evaluación más equilibrada de su producción académica y concordante con la razón de ser del conocimiento generado en este campo del saber: explicar lo que está ocurriendo en el mundo empresarial.

Finalmente, cabe advertir que concentrarse -como aquí se hizo- en los problemas que emergen del desafío de aumentar la publicación internacional, circunscritos a las tensiones específicas que plantea hacerlo en los journals top, es un abordaje muy restringido al tema. Es tanto o más importante estudiar el papel que en ese proceso pueden jugar las revistas nacionales indexadas nacional e internacionalmente, o la conformación de comunidades académicas nacionales, o la colaboración internacional para publicar (Orozco y Villaveces, 2015), o las motivaciones que guían las investigaciones -aportar a la teoría o solucionar álgidos problemas sociales o administrativos-y la originalidad de sus contribuciones. Mucho más importante es el desarrollo de las capacidades internas para investigar en las FCA, y las condiciones del entorno para apoyar ese proceso, sobre todo, el institucional, que prematuramente parece haberse desplazado del papel de promotor 
al de evaluador. Es bastante, entonces, lo que falta por conocer sobre esta coyuntura en la que se están presentando cambios vitales para el futuro de la investigación en las FCA.

\section{Referencias}

AACSB International. (2012). Impact of research. A guide for Business Schools. The Association to Advance Collegiate, Schools of Business. Tampa, Florida.

Alvesson, M., and J. Sandberg. (2013). Has management studies lost its way? Ideas for more imaginative and innovative research. Journal of Management Studies, 50 (1), 128-152.

Augier, M.; J. March, and B. Sullivan. (2005). Notes on the evolution of a research community: organization studies in Anglophone North America, 1945-2000. Organization Science, $16(1), 85-95$.

Barkema, H.; X. Chen, G. George, Y. Luo, and A. Tsui. (2015). West meets East: New concepts and theories. Academy of Management Journal, 58 (2), 460-479.

Barley, S.; G. Meyers, and D. Gash. (1988). Cultures of culture: Academics, practitioners and the pragmatics of normative. Administrative Science Quarterly, 33, 24-60.

Bartunek, J.M. (2011). What has happened to mode 2? British Journal of Management, 22, 555-558.

Bartunek, J.M., and S.L. Rynes. (2014). Academic and practitioners are alike and unlike: paradoxes of academic-practitioner relationships. Journal of Management, 40 (5), 1181-1201.

Bennis, W., and J. O'Toole. (2005). How business schools lost their way. Harvard Business Review, 83 (5), 96-104.

Calderón, G.; A. Castaño, L. García, L. y E. Castro. (2014). Generación de conocimiento en los grupos élite de investigación en Colombia. Manizales, Universidad Nacional de Colombia.

Calderón, G.; J. Arrubla, L. Gutiérrez, G. Castaño, R. Posada, A. Ruiz, A., H. Serna y J. Vivares. (2010). La investigación en administración en Colombia. Condiciones para la generación de conocimiento, investigadores, institucionalización, y producción científica. Bogotá: Ascolfa.

Coriat, B. (2000). Pensar al revés. Trabajo y organización en la empresajaponesa. México: Siglo XXI.

Corley, K. G., and D.A. Gioia. (2011). Building theory about theory building: what constitutes a theorical contribution. Academy of Management Review, 36 (1), 12-32.

Dávila, C. (1980). La crisis de la educación en Administración en Colombia. Revista Eafit Temas Administrativos, 39, 20-35.

Dávila, C. (1986). La investigación en administración: anotaciones sobre la experiencia colombiana y políticas para su promoción a nivel nacional y latinoamericano. Revista Cátedra, 1, 13-27.

Dimaggio, P., and W. Powell. (1983). The iron cage revisited: institutional isomorphism and collective rationality in organizational field. American Sociological Review, 48 (2), 147-160. 
Donaldson, L.; J. Qiu, and B. Luo. (2013). For rigour in organizational management theory research. Journal o Management Studies, 50, 128-152.

Eisenhardt, K.M. (1989). Building theories from case study research. Academy of Management Review, 14 (4), 532-550.

Eisenhardt, K.M. (1991). Better stories and better constructs: the case for rigor and comparative logic. Academy of Management Review, 16 (3), 620-627.

Eisenhardt, K.M., and M. Graebner. (2007). Theory building from cases: opportunities and challenges. Academy of Management Journal, 50 (1), 25-32.

Etzkowitz, H., and L. Leydesdorff. (2000). The dynamics of innovation: From National Systems and "Mode 2" to Triple Helix of university-industry-government relations. Research Policy, 29 (2), 109-123.

Etzkowitz, H.; A. Webster, Ch. Gebhardt, B. Regina, and C. Terra. (2000). The future of the university and the future university: Evolution of ivory tower to entrepreneurial paradigm. Research Policy, 29 (2), 313-330.

Fernández, C.J., and E. Gantman. (2011). Spain and Argentina as importers of management knowledge (1955-2008): A comparative analysis. Canadian Journal of Administrative Science, 28, 160-173.

George, G. (2014). Rethinking management scholarship. Academy of Management Journal, 57 (1), 1-6.

Gibbons, M.; C. Limoges, H. Nowotny, S. Schwartzman, P. Scott, and M. Trow. (1994). The New Production of Knowledge: The Dymnamyc of Science and Research in Contemporary Societies. Thousand Oaks, SA: Sage Publication.

Gualdrón, 0. (2016). Nuevo Publindex: Modelo clasificación de revistas científicas nacionales. Presentación en "Estado de la ciencia en Colombia". Foro Semana, Bogotá, mayo 26.

Hannan, M. and J. Freeman. (1977). The population ecology of organizations. American Journal of Sociology, 82 (5), 929-964.

Helfat, C. (2007). Stylized facts, empirical research and theory development in management. Strategic Organization, 5 (2), 185-192.

Ibarra, E. (2006). Organization studies and epistemic coloniality in Latin America: Thinking otherness from the margins. Organization, 13 (4), 463-488.

Kim, L. (1998). Crisis construction and organizational learning. Capability building in catching-up at Hyundai Motor. Organization Science, 9 (4), 506-521.

Kuhn, T. (1962). The structure of scientific revolutions. Chicago: Universidad de Chicago.

Leydesdorff, L. (2012). The triple Helix, Quadruple Helix... and N-tuple of Helices: Explanatory models for analyzing the knowledge-Based Economy. Journal of the Knowledge Economy, $3(1), 25-35$. 
López, F. (1998). Educación en administración y modas administrativas en Colombia. Revista Eafit, 109, 59-88.

Malaver, F. (2000). La investigación en gestión empresarial. Academia, Revista Latinoamericana de Administración, 23, 67-81.

Malaver, F. (2006). El despegue de la investigación colombiana en Administración: análisis de sus avances en el periodo 2000-2006. Cuadernos de Administración, 18 (32), 71-109.

Malaver, F. y M. Vargas. (2005). Las políticas de ciencia, tecnología e innovación en Colombia 1990-2005: sus logros, fracasos y desafíos. Cuadernos de Administración, 18 (30), 39-78.

Merton, R.K. (1968). The Mathew Effect in Science. Science, 159 (3810), 56-63.

Mintzberg, H. (2005). Directivos, no MBAs. Una visión crítica de la dirección de empresas y la formación empresarial. España: Universidad de Deusto.

Nelson, R. (1995). Recent evolutionary theorizing about economic change. Journal of Economic Literature, 33 (1), 48-90.

Nelson, R., and H. Pack. (1999). The Asian miracle and modern growth theory. Economic Journal, 109 (457), 416-436.

Ogliastri, E. (1982). Investigaciones en Administración. Memorias, II Encuentro de investigadores en Administración. Cali, ICFES-Universidad del Valle.

Orozco, L.A. (2015). Diversidad y heterogeneidad en redes de colaboración científica. Un estudio de las escuelas de administración de América Latina. Bogotá, Universidad Externado de Colombia.

Orozco, L.A., and J.L. Villaveces. (2015). Heterogeneus research networks in Latin American Schools of business management. Academia, Revista Latinoamericana de Administración, 28 (1), 115-134.

Pfeffer, J., and C.T. Fong. (2002). The end of business schools? Less success than meets the eye. Academy of Management Learning and Education, 1 (1), 78-95.

Rafols, I.; L. Leydesdorff, A. O'Hare, P. Nightingale, and A. Stirling. (2012). How journal rankings can suppress interdisciplinary research: A comparison between innovation studies and business \& management. Research Policy, 41, 1262-1282.

Sistema Nacional de Información de la Educación Superior - SNIES. (2016). Ministerio de Educación Nacional. Recuperado el 10 de junio de 2016 de http://snies.mineducacion.gov. co/consultasnies/programa

Stiglitz, J. (1996). Some Lesson from de East Asian miracle. World Bank Research Observer, 11 (2), 151-178.

Thomas, H., and A.D. Wilson. (2011). "Physics Envy”, cognitive legitimacy of practical relevance: Dilemmas in the evolution of management research in the UK. British Journal of Management, 22 (3), 443-456. 
Transfield, D., and K. Starkey. (1998). The nature, social organization and promotion of management research: Towards policy. British Journal of Management, 9, 341-353.

Whitley, R. (2008). Variety of knowledge and their use in business and management studies: Conditions and institutions. Organization Studies, 29 (4): 581-609.

World Bank (1993). The East Asian Miracle: Economic Growth and Public Policy. 0xford: 0xford University Press.

Yin, R. K. (2003). Case study research: Design and methods. Oaks, California, EEUU: Sage Publications.

\section{Anexo 1. Journals con mayor número de artículos publicados por autores vinculados a las FCA en el periodo 2001-2014}

\begin{tabular}{|c|c|c|c|c|c|}
\hline \multicolumn{3}{|c|}{ Journal top 20, WoS 2001-2014 } & \multicolumn{3}{|c|}{ Journal top 20, Scopus 2001-2014 } \\
\hline Journal & $\mathrm{N}^{\circ}$ & País & Journal & $\mathrm{N}^{\circ}$ & País \\
\hline $\begin{array}{l}\text { Innovar Revista de Ciencias } \\
\text { Administrativas y Sociales }\end{array}$ & 56 & Colombia & Cuadernos de Administración & 98 & Colombia \\
\hline $\begin{array}{l}\text { Academia Revista Latinoa- } \\
\text { mericana de Administración }\end{array}$ & 20 & Colombia & Innovar & 76 & Colombia \\
\hline $\begin{array}{l}\text { Journal of Business } \\
\text { Research }\end{array}$ & 12 & Estados Unidos & $\begin{array}{l}\text { Journal of Technology } \\
\text { Management and Innovation }\end{array}$ & 13 & Chile \\
\hline $\begin{array}{l}\text { European Journal of } \\
\text { Operational Research }\end{array}$ & 8 & Netherlands & Journal of Business Research & 12 & Estados Unidos \\
\hline $\begin{array}{l}\text { Systemic Practice and } \\
\text { Action Research }\end{array}$ & 7 & Estados Unidos & Academia & 9 & Colombia \\
\hline $\begin{array}{l}\text { Systems Research and } \\
\text { Behavioral Science }\end{array}$ & 5 & Reino Unido & $\begin{array}{l}\text { Systemic Practice and Action } \\
\text { Research }\end{array}$ & 7 & Estados Unidos \\
\hline Journal of Business Ethics & 5 & Netherlands & $\begin{array}{l}\text { Journal of the Operational } \\
\text { Research Society }\end{array}$ & 5 & Reino Unido \\
\hline Public Relations Review & 4 & Estados Unidos & Espacios & 5 & Venezuela \\
\hline $\begin{array}{l}\text { Journal of the operational } \\
\text { research society }\end{array}$ & 3 & Reino Unido & Journal of Business Ethics & 5 & Netherlands \\
\hline Small Business Economics & 3 & Netherlands & $\begin{array}{l}\text { Systems Research and } \\
\text { Behavioral Science }\end{array}$ & 4 & Reino Unido \\
\hline $\begin{array}{l}\text { Group Decision and } \\
\text { Negotiation }\end{array}$ & 3 & Netherlands & $\begin{array}{l}\text { International Entrepreneur- } \\
\text { ship and Management Journal }\end{array}$ & 4 & Estados Unidos \\
\hline Management Decision & 3 & Reino Unido & Cuadernos de Gestión & 4 & España \\
\hline $\begin{array}{l}\text { International } \\
\text { Entrepreneurship and } \\
\text { Management Journal }\end{array}$ & 3 & Estados Unidos & Decision Support Systems & 4 & Netherlands \\
\hline
\end{tabular}




\begin{tabular}{|c|c|c|c|c|c|}
\hline \multicolumn{3}{|c|}{ Journal top 20, WoS 2001-2014 } & \multicolumn{3}{|c|}{ Journal top 20, Scopus 2001-2014 } \\
\hline Journal & $\mathrm{N}^{\circ}$ & País & Journal & $\mathrm{N}^{\circ}$ & País \\
\hline $\begin{array}{l}\text { International Journal of } \\
\text { Manpower }\end{array}$ & 3 & Reino Unido & Public Relations Review & 4 & Estados Unidos \\
\hline $\begin{array}{l}\text { Revista Venezolana de } \\
\text { Gerencia }\end{array}$ & 3 & Venezuela & $\begin{array}{l}\text { Latin American Business } \\
\text { Review }\end{array}$ & 3 & Estados Unidos \\
\hline $\begin{array}{l}\text { International Journal of } \\
\text { Arts Management }\end{array}$ & 2 & Canada & Management Decision & 3 & Reino Unido \\
\hline $\begin{array}{l}\text { Journal of Knowledge } \\
\text { Management }\end{array}$ & 2 & Reino Unido & $\begin{array}{l}\text { International Journal of } \\
\text { Knowledge, Culture and } \\
\text { Change Management }\end{array}$ & 3 & Estados Unidos \\
\hline Research Policy & 2 & Netherlands & Utilities Policy & 3 & Reino Unido \\
\hline $\begin{array}{l}\text { Emerging markets finance } \\
\text { and trade }\end{array}$ & 2 & Estados Unidos & $\begin{array}{l}\text { International Journal of } \\
\text { Logistics Systems and } \\
\text { Management }\end{array}$ & 3 & Reino Unido \\
\hline $\begin{array}{l}\text { Review of Industrial } \\
\text { Organization }\end{array}$ & 2 & Netherlands & $\begin{array}{l}\text { Technological Forecasting } \\
\text { and Social Change }\end{array}$ & 3 & Estados Unidos \\
\hline Total & 148 & & Total & 268 & \\
\hline
\end{tabular}

Fuente: elaboración propia con base en la WoS y Scopus. 
